\title{
Tangence
}

\section{Parler le Livre}

\section{Dans l'enseignement, le lieu d'un transfert en triangle}

\section{Francine Belle-Isle}

Numéro 54, mars 1997

Poétique du Livre

URI : https://id.erudit.org/iderudit/025933ar

DOI : https://doi.org/10.7202/025933ar

Aller au sommaire du numéro

Éditeur(s)

Tangence

ISSN

0226-9554 (imprimé)

1710-0305 (numérique)

Découvrir la revue

Citer cet article

Belle-Isle, F. (1997). Parler le Livre : dans l'enseignement, le lieu d'un transfert en triangle. Tangence, (54), 7-17. https://doi.org/10.7202/025933ar d'utilisation que vous pouvez consulter en ligne.

https://apropos.erudit.org/fr/usagers/politique-dutilisation/ 


\section{Parler le Livre. Dans l'enseignement, le lieu d'un transfert en triangle}

Francine Belle-Isle

Jamais, dans l'exercice concret de la théorie analytique, nous ne pouvons nous passer diune notion du manque de l'objet comme central. Ce n'est pás un négatif, mais le ressort même de la relation du sujet au monde.

J. Lacan, La relation dobjet

Ainsi cependant vous avez pu vivie cet amour de la seule façon qui puisse se faire pour vous, en le perdant avant qu'il soit advenu.

M. Duras, La maladie de la mort

Parler le Livre, directement et sans filet, comme on fait ouverture à l'Autre inconnu, et non parler du livre, sagement, savamment, d'assez loin pour qu'il ne risque pas de compromettre un dire de suffisance. Pour une fois, cesser d'être hypocrite et accepter que la parole professorale, celle mise à l'épreuve du texte littéraire, doive elle aussi rencontrer les servitudes de son désil: Cependant, qu'il soit d'entrée de jeu posé que je veux parler ici d'un certain enseignement, de l'enseignement vu cl'une certaine façon, dans ses détours intimes je dirais, là où cependant il s'agit, paradoxalement, bien plus des positions respectives des actants, voire de leurs postures, que de leurs qualités personnelles. Avec tous les risques d'impureté que cela impute à la réflexion et que pourraient me reprocher sans doute quelques tenants des sciences de l'éducation. Je sais le danger qu'il y a à penser l'espace pédagogique, du moins celui qui prend à sa charge la parole du Livre, comme le lieu d'un théâtre, où les audaces de la mise en scène seraient presque toutes à mettre au compte des fantaisies du transfert. C'est, de fait, supposer un toujours probable désordre dans la transmission du savoir, briser la belle ligne droite de l'autorité magistrale, et semer la pagaille dans ce qui devrait 
8

s'ordonner d'une stricte hiérarchie. C'est aussi, et surtout, pour qui parle d'une parole d'enseignement, se remettre radicalement en question comme diseur de vérité. On aimerait sans doute mieux voir les choses autrement et pouvoir se replier sur un détachement de circonstance, quand les certitudes de la connaissance devraient suffire à rassurer et à garantir un discours de tout repos. Mais, comme Serge Leclaire à propos de l'amour de Sygne, *je ne crois pas à l'illusion neutralisante du masque impassible, et ne trouve, en l'occurrence, aucune nécessité à me défendre de ce qui pourrait m'être imputé au titre de la séduction. ${ }^{\text {. Un peu }}$ d'hystérie, surtout quand elle trouve un rôle à sa mesure et peut jouer serré, n'a jamais fait de mal à personne. À condition toutefois que reste vive et inquiète cette interrogation, seule à valoir le prix d'un possible silence: "quand j'enseigne, d'où me vient la parole et où va-t-elle?.

Ici, on le comprend, c'est bien le lieu de la profération, comme limite d'espace et de temps consentie à une expérience particulière, celle des mots donnés en pâture, qui devrait compter plus que tout et moduler seul les accents de la voix, et non pas la leçon apprișe par coeur par les répétiteurs de service qui toujours sommeillent en nous et qui s'avisent tout à coup de prendre au sérieux leur savoir en osant l'imposer comme des vérités de La Palice à des écoutants dès lors castrés de tout désir et rendus muets par la force des choses. Si la pratique de la psychanalyse, comme épreuve du libre champ de la parole, n'apprend pas à professer le texte littéraire autrement que comme le signe bête et figé d'une référence toute entière déposée là, telle qu'en ellemême l'éternité la trouve, ça ne vaut pas la peine de revendiquer le droit à une légitimité critique dont on s'emploie, par ailleurs, à invalider le sens. À partir du moment où l'on prétend savoir qu'il n'y a d'objet que dans sa quête, de désir que dans le report de la demande, de jouissance vraiment humaine que dans les tissures du langage, il faut bien en accepter les conséquences, toutes les conséquences, dont l'essentielle consiste, me semble-t-il, à prendre sur soi le poids de la parole, celle du texte mêlée à la sienne, et l'inverse aussi, et à porter en connaissance de cause et à la force de ses mots la charge de son propre transfert, pour que se fasse dans l'enseignement la rencontre souhaitée avec le trans-

1 Serge Leclaire, On tue un enfant, Paris, Seuil, coll. * Le champ freudien. 1975, p. 97. 
fert de l'autre, l'autre écoutant et ne sachant pas, lui, à quels échos il est en train d'arrimer sa parole silencieuse.

On dira peut-être que je prends bien facilement mon parti du transfert et que, loin de m'en méfier et de vouloir le tenir en respect dans la lecture enseignée du texte littéraire, je lui fais une place de choix. Il me semble qu'il n'est pas absolument souhaitable de repasser par toutes les émotions contradictoires qu'a connues Freud à son sujet et que, devant son inéluctable destin, mieux vaut essayer tout de suite de s'en arranger, à défaut de quoi on risque la catastrophe. La catastrophe étant ici la méconnaissance absolue des lois qui régissent la parole "publique", et qui l'obligent toujours au bout du compte à se répercuter sur l'écran d'un désir mis en un lieu commun, ce qui ne veut pas dire nécessairement ou unanimement partagé. Autrement dit et plus crûment, je ne pense pas qu'on puisse passer de la lecture privée, celle dont le face à face se fait avec le texte seul en une sorte de méditation silencieuse, à une lecture publique, celle déclamée de la chaire d'enseignement, sans que quelque chose d'autre, de l'autre, ne vienne s'adjoindre dans la parole et la déporter en quelque ailleurs insu.

Je n'irais sans doute pas jusqu'à affirmer que le bon professeur, c'est celui qui ne sait pas ce qu'il dit, le disant, mais c'est certainement celui qui sait ne pas pouvoir savoir tout ce qu'il va dire, l'assumant. Que la mise au jeu de cette part transférentielle l'amène là où sa parole n'avait pas prévu d'aller et qu'il soit tenu de reconnaître ce dire-là aussi et peut-être surtout comme l'expression de son désir propre sur le texte n'est pas sans modifier ses acquis de lecture, sans disposer autrement son regard, et donc sans traverser son enseignement d'une mesure d'inouï et d'inédit. L'écoutant, lui, ne se méprend jamais sur ce qu'il lui est donné d'entendre pour la première fois comme ça, forcément donc comme l'aveu d'un secret partagé dans la grâce de sa présence, et reçoit alors la parole professorale comme le signe vivant d'une intimité que seuls les mots ont charge de soutenir. Convoqué sur la scène parlée de la lecture de l'autre dont il devient le partenaire imaginaire, son narrataire en miroir, l'écoutant qui est là pour apprendre à lire un texte se voit témoin d'une expérience en train de se faire, complice en direct de ce que l'interprétation n'est pas un jugement arrêté dans l'avant-coup d'une théorisation catégorique, mais bien l'intrusion du sens là où celui-ci ne pensait pas avoir affaire, et sa persistance à vouloir y demeurer. Il 
comprend, il faut le souhaiter, que lire un texte n'a plus grand chose à voir avec l'accusé de réception d'une lettre déjà écrite en sa fin, et que c'est seulement dans la prise des mots de l'autre que se construit, ici et maintenant, le sens dit d'un corps de fiction. Montrer ainsi la vulnérabilité de l'interprétation, ou plutôt sa possible suspension dans le temps de son émergence, et les avenues subjectives qui en règlent aussi le parcours, est-ce déplacer indûment les enjeux d'un enseignement autorisé et donner prise à ceux qui tiennent la chose littéraire comme le lieu du sentimental et de l'évanescent? Je ne le crois pas, quand cette place faite à l'impensé s'accompagne de l'assurance formelle de pouvoir en assumer la charge dans le temps et l'espace pédagogiques destinés précisément à y penser. Que cela exige une compétence à toute épreuve, dont toutes les armes doivent être sans cesse affûtées, l'essentielle étant un préjugé inconditionnellement favorable à ce qui reste à découvrir, cela ne fait pas de doute et confère à la parole professorale le devoir de rendre des comptes aussi sur ce qu'elle ne sait pas bien encore. L'obligation lui est donnée en quelque sorte d'improviser sur-le-champ le savoir de son désir, aussitôt que celui-ci vient toucher le texte à lire d'un sens nouveau et déjà senti comme indispensable. La véritable performance, la seule intéressante au fond pour qui enseigne avec la psychanalyse, consiste donc à reconduire, sous la forme d'une impeccable cohérence et dans l'instant même de la parole, ce désir de la vérité du texte en train d'advenir à lui-même.

De la capacité de symboliser pour soi et pour l'autre, et au fur et à mesure qu'elles apparaissent, les lucidités de lecture apportées par les impératifs transférentiels dépendent directement les forces de séduction de l'appareil interprétatif, celui-ci passé au crible d'une écoute dont les intérêts eux aussi participent d'une approche affectivement investie. Avant d'essayer de cécrire les détours et particularités de cette composante réciproque, il convient d'abord d'en tenir compte comme contrainte de la réalité pédagogique et de voir quelles conséquences elle entraine dans l'exposition de la parole professorale. Déjà dévoyée hors des sentiers battus par la réflexion solitaire et muette, à l'évidence destinée à être entendue, fût-ce par elle-même, celle-ci ne peut guère se contenter de résonner dans l'éclat d'une première fois. Elle appelle le recommencement et la reprise, dans la mise en abyme parfaite de son caractère transférentiel, ne pouvant pas voir dans sa répétition insistante autre chose que le signe toujours infor- 
mulé de son désir, un peu comme si à reprendre sans cesse la mise en place du sens celui-ci ne risquait plus de se laisser oublier. Quand Barthes, dans Le bruissement de la langue, signale que la parole d'enseignement ne souffre pas l'effacement, qu'elle se pose sur les choses du monde comme grave et définitive, j'ai souvent pensé qu'il sous-entendait là précisément sa valeur transférentielle et la reconnaissait comme l'inscription d'une marque irréversible de signification, c'est-à-dire comme une profération indéfiniment réitérée, quel que soit par ailleurs l'objet immédiat de sa quête. C'est, me semble-t-il, cette insistance du désir à investir le texte des traces repérables de sa capture qui indétermine les limites de ce qu'on a coutume d'appeler la patience de cette parole, quand elle accepte soi-disant avec générosité de reprendre inlassablement les fils d'une explication mal comprise. C'est bien plus de passion que de patience qu'il s'agit ici, alors que le désir de voir reconnu son amour du texte risque d'être trahi par les incompétences mal venues de qui est là justement pour le satisfaire. Et c'est toujours la passion ignorée de cette parole, avec la privation qui l'accompagne, qui fait retour dans l'éclatement de l'impatience dont les retombées égocentriques ne font pas de doute. On comprend alors un peu mieux de quoi est faite l'endurance du professeur qui, année après année, s'expose encore à devoir trouver les mots justes pour parler de ce qu'il continue d'ignorer, sinon dans l'acte de parole qui le met en scène et le place dans la périlleuse position du supposé tout savoir, quand il n'est pas sûr du tout que les écoutants du moment pourront recevoir sa lecture comme l'exacte mesure de leur propre demande.

Car il faut bien finir par poser la question. La question de leur désir, à eux, les écoutants. Parce qu'il n'est pas du tout sûr que ces autres à qui se donne la parole du maître, et dont celui-ci accepterait sans doute qu'ils puissent la refuser pour préférer la leur, soient de quelque façon au courant de ce qui l'agite, je ne dis pas à la rencontre de son désir mais au moins dans l'écoute de son mouvement même. Il n'est donc pas simple d'avoir ainsi à exposer à l'Autre sa passion, à lui dévoiler ses investissements à fonds perdu, à donner en pâture sa folie à qui se trouve là pour voir, surtout quand ce quelqu'un d'autre se représente de quelque quatre-vingts paires d'yeux dardés sur soi, pas choisis, donnés plutôt comme des juges, au mieux indulgents au pire intraitables. À ces affamés souvent de recettes et de trucs, de tous 
les faux-fuyants commodes qu'il conviendrait d'inventer pour leur faire croire que parler le Livre c'est faire illusion de ce qu'il soit devenu sa chose, sans les mesures compensatoires qu'à tout le moins cela exige, il est difficile de résister, de persister à offrí une parole inquiète, provisoire, qui s'entête à chercher encore ce qui paraît se donner comme infiniment probable. Pourtant c'est bien cette persévérance-là qui seule peut garder intact leur désir du Livre encore fragile, et les assurer qu'il n'y a d'amour que du lieu d'un manque.

Amour de transfert, bien sûr, mais incontestablement véritable affaire d'amour, pour qui ne méconnaît pas la dimension excentrique de l'amour, celle qui le fait rapport d'insuffisance réciproque et qui le reste, dans le don à l'autre de cette insuffisance comme cause et garantie de désir partagé. Si *aimer, c'est donner ce qu'on n'a pas à quelqu'un qui n'en veut pas", formule Lacan, cela doit s'entendre non pas à imaginer la relation privilégiée des êtres de langage comme le partage d'un bien-être de consommation, mais plutôt à consentir à l'insensé que suppose toujours l'échange amoureux, au défaut d'être fondamental qui est celui d'un couple sujet-objet, l'un à l'autre jamais-vraiment-làpour-vrai-et-pour-de-bon, chacun donné et retenu pour la relance même du désir. C'est dire que la parole professorale, en tant que parole doublement amoureuse, amoureuse du Livre à lire et amoureuse de qui est là pour recevoir cette lecture, ne peut venir que d'un corps d'enseignement nulle part fixé, insituable, atopi$q u e$, seul capable de faire se représenter dans le jeu du signifiant le fort-da du sens, quand dans l'écart symbolique de la signification finit quand même par advenir, sorte de présence-absence d'une vérité désormais interdite. C'est dire aussi qu'en tant que voix discourant dans l'ombre d'une perte essentielle, la parole professorale devrait s'écouter à l'épreuve de son deuil, dans l'urgence d'inventer ailleurs que dans sa peine la reprise réparatrice d'un verbe en faillite. Signe reconduit d'un désir impossible, la voix du maitre, si elle n'en ressort pas moins, dans l'éclat de sa fonction phallique, d'un crédit aussi brillant qu'éphémère, ne saurait donc s'en prévaloir que du lieu même de sa déficience, de là où s'entendent encore les accents d'une insistance à prendre en compte la barre qui marque toute énonciation dans son rapport à la Chose innommable. Ainsi circonscrite dans le champ de sa propre quête, en constante dérive par rapport à ce qu'elle construit, la parole professorale a l'obligation éthique de témoigner, 
non seulement de ses acquis de connaissance, mais aussi de tous les efforts consentis pour désavouer son ignorance, c'est-à-dire pour la reconnaître là même où elle apparaît magistralement déniée.

Dans cette rencontre toujours ratée avec le Livre, l'enseignement du texte littéraire devient sujet de passion, c'est-à-dire lieu choisi pour déposer là en souffrance un objet d'inépuisable connaissance. Sorte d'objet $a$, ici bien sûr identifié et représentable, mais seulement dans la chute de ses présentations, dans les * semblants " de sa représentation. Si le concept de littérarité a tant de mal à trouver sa définition autrement que dans des approximations plus ou moins satisfaisantes, c'est bien parce que l'objet qu'il recouvre, qu'il voile plutôt qu'il ne le découvre, échappe à toute saisie définitive, n'offrant à qui s'en aveugle que les leurres de ses multiples métamorphoses. Que dans le fantasme, l'objet $a$ fasse éclater ses lieux d'identité en quatre morceaux détachés du corps - sein, fèces, regard et voix - est loin d'être sans importance, puisque comme par hasard se trouvent ici nommés les quatre constituants du Livre, aussi de toute ceuvre d'art: sa genèse (sein), sa production (fèces), sa réception (regard) et sa promotion (voix). Comment donc la voix du maitre, précisément, pourrait-elle accepter de trahir son objet et de se trahir elle-mếme en refusant de montrer que le sujet de sa passion est en même temps le lieu de son deuil?

Mais, ne soyons pas dupes, même assourdie dans ses certitudes et s'adressant à une ignorance présumée, la parole du maître rencontre toujours de fait le savoir indu de l'écoutant. Car dès que celui-ci entend ce qu'il saisit comme retour de son attente et donc comme allusion directe à son désir, il est mis nécessairement déjà dans le champ de son savoir. Je le rappelle, il est question ici de positions pédagogiques, et non des personnes légitimes qui les occupent. Cela entraîne parfois des aberrations, par les jeux de coulisses qui risquent de transformer précisément ces personnes en autre chose qu'elles-mêmes, simplement en inversant la dynamique pédagogique par exemple: en faisant de l'écoute le lieu d'un savoir instantané, qui surgit dans le temps même où elle vampirise la parole du maître, et où celle-ci vient s'éprouver dès lors comme le reste d'elle-même, écho fragile de ce qui semble devenu tout à coup propriété de l'autre et faire de soi un cadavre vidé de toute connaissance. C'est méconnaitre la réalité que de penser le métier de professeur comme tellement de 
l'ordre de la dévotion qu'il ne peut déboucher que sur l'euphorie de la transmission du savoir, en oubliant de voir qu'il y a aussi effet d'effraction et de perte quand, dans le tournant des choses, l'écoute de la parole professorale devient aussi le temps de son effritement et de sa dispersion. Peut-être faut-il cependant remettre l'affaire dans sa juste perspective et se consoler de ce qu'il y a probablement là l'occasion d'une découverte importante à faire, à savoir peser la différence essentielle que les positions du maître et de l'écoutant supposent dans leur rapport au savoir et la considérer, cette différence, comme devant structurer absolument la relation pédagogique, faute de quoi quelque chose fatalement se perd de l'expérience de la connaissance. S'il arrivait que l'objet du savoir, le Livre en l'occurrence, et ce ne peut être qu'un livre - je parle ici d'une auvre littéraire —, n'ait pas même valeur pour celui qui professe que pour celui qui écoute, s'il ne se représentait pas de la même fonction pour l'un et pour l'autre, je crois que cela pourrait expliquer et impliquer pas mal de choses.

Je fais donc l'hypothèse que le texte à lire est toujours, pour le maître, de l'ordre du réel et, pour l'étudiant, de l'ordre de l'imaginaire, étant entendu que le point de jonction - la transmission du savoir - doive s'accomplir nécessairement dans l'espace symbolique. Que les déplacements transférentiels viennent toujours aussi bouleverser cet équilibre triangulaire, il faut évidemment en tenir compte, mais ne pas en perdre de vue la dynamique fondamentale. La parole professorale est bien alors cette parole impossible, sur un texte perdu pour l'entendement, dont il faut pourtant porter la trace dans des mots impuissants à révéler l'origine du sens autrement que dans l'effort consenti pour en faire se répercuter l'écho. Parole arrachée donc à une ignorance essentielle, mais qui refuse de faire le deuil cle son savoir et qui l'invente sur fond de leurre et de simulacre. Ce qui explique sans doute pourquoi, à un certain point de vue, on ne sait bien que ce qu'on enseigne, parce qu'on sait aussi du lieu de quelle perte on paie cet exploit. Il peut arriver bien sûr qu'on oublie, dans le feu d'un verbe autoritaire, la part de méprise qui le traverse, quand par exemple la tentation de se prendre pour la lettre du texte finit par suturer la faille dans le savoir et déporter la parole professorale vers une maîtrise qui ne saurait être la sienne. Mais il reste que c'est là un dérapage insensé et qu'il faudra bien, tôt ou tard, que cette parole faussement magistrale frappe le mur, 
se prenne à bafouiller et retrouve l'humble chemin de ses rêves. Et c'est peut-être cela finalement, cet emprunt du symbolique à la vérité perdue, dont l'écoutant fait l'expérience aveugle quand il saisit au vol la parole qui en témoigne, là devant lui, et qu'il la reçoit spontanément pour ce qu'elle est, l'évocation d'un sens aliéné, qui n'appartient plus à personne, pas même à celui qui le profère. Pour peu qu'il se sente touchê par elle, en quelque point que ce soit, cette parole il va s'en emparer, du registre qui est le sien, celui de l'identification et de l'imaginaire, et en disposer comme de son bien propre, la reprenant à son compte, l'ajustant à son désir du texte, jusqu'à ce qu'elle lui aille comme un gant et qu'il puisse la comprendre comme sa propre lecture, comme la seule lecture possible pour lui dans le champ de ses intérêts et de ses compétences.

La parole professorale aurait donc pour l'écoutant effet d'image, dans laquelle il se mire, reconnaissant dans cette voix autre la seule qui puisse devenir la sienne et s'y accrochant comme au signe même de son savoir à venir. Que cette fascination captatrice qui le fait basculer dans le désir de l'autre soit aussi, en même temps qu'elle le pose dans son rôle d'enseigné, la voie royale qui mène à sa dépendance et à sa soumission, c'est là certainement une affaire entendue dont il devra trouver moyen de s'arranger, s'il ne veut pas découvrir tout à coup, pour sa désillusion, de quels vains mirages participent ses nouvelles certitudes. Mais pour peu que la parole professorale, séduite par les complaisances de l'imaginaire, ne se presse pas de briser le miroir et laisse s'installer les plaisirs de la mimésis, la jubilation spéculaire risque de durer longtemps et de devenir même le modèle légitime d'une pédagogie réussie. Il est évident qu'il peut y avoir des bénéfices immédiats à profiter de l'espèce d'engourdissement plus ou moins admiratif qui tient l'écoutant dans une filiation docile et le fait dépositaire d'un savoir programmé. Cela est sans doute nécessaire même, quand il faut assurer la pérennité d'un héritage de connaissances, mais dans l'espace d'enseignement du Livre, là où il s'agit de parler la parole, donc de doubler la mise symbolique, je ne vois pas trop bien comment éviter éternellement de faire l'épreuve du signifiant, refuser d'en accepter la nature fantasque et foncièrement autonome et nier du même coup l'économie essentiellement subjective qui l'organise en sa trame. Dans cet ordre des choses, il y va d'une parole professorale responsable de rompre les charmes de l'imaginaire et de 
ramener la lecture publique du texte là où elle sait qu'elle doit se trouver: au champ du symbolique, dans ce registre qui marque l'objet de parole de tous les signes de l'absence et fait de sa fragile possession le lieu déjà d'une rupture.

L'équivoque n'est pas permise. De quelque façon que cela se fasse, il faut qu'il y ait, dans un enseignement de ce type, introduction au désir du texte, tel qu'essaie d'y advenir elle-même la parole de qui tient la position du maitre. Parole qui doit porter la trace d'une attente et aussi d'un élan qui en calme l'ardeur, avec toutes les vicissitudes d'un transfert qui consent à ne jamais voir réponse adéquate à sa demande et qui, pourtant, ne renonce pas à déchiffrer les termes de son espérance. Ce n'est qu'affronté, toujours, mots après mots, à cette parole de désir que l'écoutant peut arriver à renoncer à son rêve de possession tranquille pour prendre, lui aussi, le risque du réel que lui représente sans défaillance la voix du maître. Toute pédagogie qui se respecte sait bien qu'il n'y a pas d'enseignement efficace si celui-ci n'arrive pas à communiquer le désir de l'objet, le problème c'est que trop souvent elle entend le combler, ce désir, comme la récompense due à l'acceptation volontaire du contrat d'apprentissage. Cette mentalité de mercenaires, comme je l'ai déjà appelée, est fatale absolument ici, quand la voix qui lit le texte cherche, au contraire, à mettre dans l'oreille de l'écoutant l'accent d'une inquiétude, la sienne devant les insuffisances de sa prise, et à lui faire entendre qu'il n'est de sens que dans l'éclat d'une parole disséminée. À ceux qui s'inquièteraient encore de l'affiche d'une pareille vulnérabilité dans une pratique de parole qui doit demeurer l'exercice d'une compétence, je dirais que cette vulnérabilité-là ne peut se faire sentir que dans la compétence, elle y est en quelque sorte inscrite comme sa preuve la plus vive, quand dans l'assurance d'une démonstration, incontestable de pertinence, s'écoute quand même la fêlure d'une hésitation, le temps d'une syncope à peine marquée, qui rappelle la mélancolie d'un verbe solitaire.

Quand Barthes toujours, dans $S / Z$ cette fois, dit que "la version première d'une lecture doit pouvoir être sa version dernière " ${ }^{2}$, il me semble que si cela a des chances d'être vrai, c'est justement dans la lecture publique, professorale surtout, qui passe obligatoirement par le crible de la voix comme par l'épreuve

2 Roland Barthes, $S Z$, Paris, Seuil, coll. - Points ", 1976, p. 22. 
même de sa résistance, sans autre alternative que les multiples possibles qu'elle lève au devant d'elle, de la même façon que se lève le gibier qu'on va ensuite traquer comme la proie donnée à sa capture. Lecture toujours première, parce qu'on ne se souvient plus de l'avoir faite avant l'instant de sa profération, mais dernière aussi, toujours, parce qu'on y est indissolublement lié, clans la quête éperdue qu'elle suppose et à laquelle la fin de la séance d'enseignement n'arrivera qu'à mettre un suspens provisoire. Aussi dans un article qu'il a écrit sur City of Glass de Paul Auster et qu'il titre * Une Babel en verre dépoli n, Bertrand Gervais souligne-t-il cette expérience d'une lecture interminable, pour lui privée, mais à laquelle il donne une résonance exemplaire:

C'est cela peut-être qui explique ma relecture, cette posture
dans laquelle je me maintiens et dont je ne retrouve plus l'ori-
gine. [...] Je la reprends donc sans cesse, je l'habite, je fais un
avec elle, comme s'il n'y avait plus rien entre les lettres et moi
et que nous étions, nous aussi, irréversiblement liés. Je ne peux
m'imaginer maintenant ne jamais avoir lu le roman, c'est pour
cela que je ne sais plus rien de ma lecture première. Elle m'est
tout aussi clifficile à imaginer que la toute dernière qui me ferait
m'évanouir.

Lorsque la parole professorale réussit à reprendre à son compte quelque chose de cette exacerbation à lire personnellement un texte dont par ailleurs les limites à franchir dépassent l'entendement, il arrive que l'écoutant - jusque-là attentif à prendre la dictée en des notes qu'il veut les plus fidèles possible, quitte à faire répéter ce qui ne cesse de l'être, et comme si pouvait exister une mémoire de la voix - lève la tête, dépose le crayon et prenne le temps de regarder cette voix et d'y reconnấtre tout à coup l'annonce voilée de sa prochaine errance.

Étant entendu depuis le début que cette scène-là ne peut se jouer qu'à l'intérieur d'une certitude, que le réel en question ici, s'il n'est jamais réductible au symbolique, y est cependant lié comme son ombre et que ce n'est donc pas parce que les choses se dérobent qu'il faut pour cela renoncer aux signes.

3 Bertrand Gervais, - Une Babel en verre dépoli •, Tangence, n" 43, mars 1994, p. 23. 